\title{
Evaluación de un biorecubrimiento comestible a base de almidon de ñame modificado
}

\author{
Evaluation of an biofilms edible \\ starch-based yam modified
}

\begin{abstract}
Coatings are defined as edible products which form a thin layer on the food, and are characterized by it constitute a semipermeable barrier to gases and water vapor retarding food spoilage, improve the mechanical properties, help maintain the structural integrity of the product wrapping, to retain volatile compounds and can act as a vehicle for food additives. We evaluated the performance of the biofilms on melon through the determination of physico-chemical and sensory properties. The results show that all the variables are significantly influenced by the biomolecule employed "modified starch", noting a favorable performance in the edible biofilms. Key words: Biofilms, Cucumis melo L, modified starch, dodecenyl succinic anhydride.
\end{abstract}

\section{INTRODUCCIÓN}

El ritmo de vida de la sociedad actual ha multiplicado la demanda de alimentos de consumo fácil, rápido y seguro, como es el caso de las frutas mínimamente procesadas. Estos productos Ilamados de cuarta gama, tienen como principal desventaja que son más susceptibles al deterioro que cuando el fruto está entero, como es el caso del melón (Cucumis melo L.) que se vuelve más perecedero cuando es sometido a un procesado mínimo, por lo que se buscan alternativas para mejorar la calidad de este tipo de productos $(1,2)$. Actualmente se han desarrollado tecnologías tendientes a alargar el tiempo de conservación de este tipo de productos, dentro de estas opciones se encuentran los recubrimientos comestibles, los cuales proporcionan una cubierta protectora adicional cuyo impacto tecnológico es equivalente al de una atmósfera modificada, por lo tanto representan una alternativa a este tipo de almacenamiento, ya que es posible reducir la cinética de los cambios de calidad y pérdidas en cantidad (3).
Miladys Torrenegra A. $(1,2)$

Glicerio León M. $(1,3)$

German Matiz M. (4)

Nerlis Pájaro C. (5)

Judith Sastoque G. (1)

(1) Grupo de Investigación de Biotecnología e Innovación -GIBEI Centro de Comercio y Servicios, Regional Bolívar. SENA. Cartagena, Colombia. (2) Grupo de Investigación en Ingeniería, Innovación, Calidad Alimentaria y Salud-INCAS-, Facultad de Ingeniería, Universidad de Cartagena. Cartagena, Colombia. (3) Grupo de Investigación en Tecnología Farmacéutica Cosmética y de Alimentos -GITFCA-, Facultad de Ciencias Farmacéuticas, Universidad de Cartagena. Cartagena, Colombia.

(4) Grupo de Investigación en Tecnología de Productos Naturales - TECPRONA Facultad de Ciencias, Universidad Nacional de Colombia. Bogotá, Colombia. (5) Grupo de Ciencias Médicas y Farmacéuticas. Facultad de Ciencias de la Salud, Universidad de Sucre. Sincelejo, Colombia.

Dirigir la correspondencia a:
Profesora Miladys Torrenegra A.
Grupo de Investigación de Biotecnología e Innovación -GIBEI,
Centro de Comercio y Servicios, Regional Bolívar. SENA.
Cartagena, Colombia.
E-mail: mtorrenegraa@sena.edu.co

Este trabajo fue recibido el 13 de Octubre de 2015 aceptado con modificaciones el 3 de Abril de 2016 y aceptado para ser publicado el 2 de Julio de 2016.

Por lo anterior, se hace necesario realizar investigaciones enmarcadas en la temática de recubrimientos comestibles para evitar los daños que produce el procesado mínimo de las frutas como el melón. Una gran opción para formular un recubrimiento comestible para este tipo de producto de IV gama es el almidón de ñame de espino (Dioscorea rotundata L.), que es un tubérculo autóctono de la región Caribe Colombiana. El objetivo de este estudio fue evaluar el efecto de un biorecubrimiento comestible con base en almidón modificado (docecenil-succinatado) de ñame en melón mínimamente procesado.

\section{MÉTODOS}

El melón (Cucumis melo L.) fue recolectado en el municipio de Turbaco, ubicado en el norte del departamento de Bolívar (10 19' 30" latitud norte y $1^{\circ}$ 17' 29" longitud oeste). El biopolimero, almidón succinatado de ñame empleado fue tomado de una muestra almacenada en el laboratorio del grupo 
de investigación en tecnología farmacéutica, cosmética y de alimentos de la universidad de Cartagena. (Las condiciones de almacenamiento para el almidón modificado fue de $20 \pm$ $5{ }^{\circ} \mathrm{C}$ y HR del $\left.60 \%\right)$.

\section{Procesamiento del melón}

Los melones se seleccionaron teniendo en cuenta que estuvieran libres de daños externos y presentaran madurez comercial; se lavaron y desinfectaron con citrosan a 2,5 ppm, posteriormente se trocearon con un peso promedio de $100 \mathrm{~g}$ y se sumergieron en una solución de $\mathrm{CaCl} 2$ al $1 \%$ durante 5 minutos, secándose a temperatura ambiente; se empacaron en bolsas herméticas y posteriormente se mantuvieron a una temperatura de $4{ }^{\circ} \mathrm{C}$ (4).

\section{Análisis químicos}

Las muestras de pulpa de melón se homogenizaron y se les realizaron diversas pruebas. Inicialmente se determinó el valor del pH según el método de la AOAC 10.041/84 (5), seguido de la medición de los sólidos solubles con refractómetro (Sper Scientific, Estados Unidos). La acidez titulable expresada en $\%$ de ácido cítrico, se evaluó de acuerdo a la Norma Técnica Colombiana NTC 5208 (6-8).

Elaboración y aplicación de los biorecubrimientos (RC)

La solución base para elaborar los recubrimientos compuestos fue almidón succinatado al $4 \%$ disuelto en glicerina al $3 \%$. Cada biopelícula se elaboró mezclando los diferentes componentes (cada uno en solución individual) según el método de vaciado en placa (casting) descrito por Vázquez-Ovando et al. (1). Como agente antimicrobiano para las soluciones se empleó aceite esencial de tomillo $8 \mu \mathrm{g} / \mathrm{mL}$.

$\mathrm{T}_{0}$ : Blanco. Muestra sin recubrimiento.

$\mathrm{T}_{1}$ : recubrimiento a base de almidón de ñame succinatado (5 $\mathrm{mL}$ de anhidro dodecenil succínico DDSA) al $4 \%$ de concentración en el recubrimiento + glicerol al 3\% + aceite esencial de tomillo a $8 \mu \mathrm{g} / \mathrm{mL}$.

$\mathrm{T}_{2}$ : recubrimiento a base de almidón de ñame succinatado (10 $\mathrm{mL}$ de anhidro dodecenil succínico DDSA) al 4\% de concentración en el recubrimiento + glicerol al 3\% + aceite esencial de tomillo a $8 \mu \mathrm{g} / \mathrm{mL}$.

\section{Aplicación como RC en frutos}

La aplicación de los RC se realizó de forma manual empleando el método de inmersión. Después de la aplicación de los RC los frutos se mantuvieron a $5 \pm 1{ }^{\circ} \mathrm{C}$ y $85 \%$ HR por $15 \mathrm{~d}(1)$.

\section{Pérdida de peso (\%PP)}

Con el fin de determinar la pérdida fisiológica de peso, se realizó un seguimiento a los frutos recubiertos durante 15 días, pesando las muestras cada tres días, cuantificando el peso inicial y peso final. Los resultados obtenidos se evaluaron utilizando la ecuación 1 y se expresaron como porcentaje de pérdida de peso de la fruta (\%PP) $(1,2)$.

$$
\% \mathrm{PP}=\mathrm{Po}-\mathrm{Pf} / \mathrm{Po} \times 100 \quad \text { (ecuación 1) }
$$

Donde, \% PP es el porcentaje de pérdida de peso, P0 es el peso inicial de la muestra, Pf es el peso final de la muestra.

\section{Análisis sensoria}

Para evaluar las propiedades organolépticas de los productos recubiertos, se realizó un análisis sensorial en un panel conformado por estudiantes de último semestre del programa de Ingeniería de Alimentos. Estaba compuesto por cuarenta panelistas no entrenados. La evaluación de los RC fue realizada mediante una escala hedónica de 6 puntos: aceptabilidad, donde 1 (me disgusta mucho), 2 (me disgusta), 3 (ni me gusta ni me disgusta), 4 (me gusta ligeramente), 5 (me gusta), 6 (me gusta mucho) (1).

\section{Análisis estadístico}

Los resultados correspondientes a tres ensayos independientes se expresaron como el promedio \pm el error estándar de la media (ESM) y se analizaron mediante pruebas t y análisis de varianza de una vía (ANOVA), seguido de prueba de Dunnet o Tukey post hoc para comparaciones múltiples. Valores de $\mathrm{P}$ $<0,05$ fueron considerados significativos. Para la organización de los datos se empleó la hoja de cálculo MS Excel 2010, y para los análisis estadísticos el paquete GraphPad Prism V5.00 para Windows.

\section{RESULTADOS}

La calidad de un fruto contempla además de sus características físicas -tamaño, peso, color y textura- su contenido nutricional, expresado en sólidos solubles totales, acidez y nutrientes (5). En la tabla 1, se presentan los resultados de la caracterización de análisis químico de la pulpa de melón.

La variación del $\mathrm{pH}$ resultó significativa, la tendencia mostrada en la tabla 2, puede ser consecuencia del stress al cual se somete el fruto, ya que una vez arrancado de la planta durante los primeros días se ve obligado a gastar parte de sus ácidos orgánicos como parte de sus procesos metabólicos (2).

Para los diferentes recubrimientos no se encontraron diferencias significativas, pero sí para las variaciones de la propiedad durante el tiempo (tabla 3 ).

La aplicación de los recubrimientos en los melones generó diferencias significativas en el contenido de sólidos solubles totales entre los tratamientos evaluados durante el almacenamiento ( $p>0.05)$, como se observa en la tabla 4.

El tratamiento estadístico realizado a los datos obtenidos en el ensayo de pérdida de peso indicó que hay diferencias significativas en la pérdida de peso para cada uno de los fac-

\section{TABLA 1}

Caracterización física de la pulpa de melón (Cucumis melo L.)

Parámetros evaluados

$$
\mathrm{pH}
$$

Acidez
Resultados

$5,80 \pm 0,01$

$0,10 \pm 0,00$

$9,41 \pm 0,08$

Solidos solubles totales (SST) 
tores evaluados (tabla 5).

En la figura 1 se encuentran los resultados de la prueba sensorial que se realizó para determinar si existían aceptabilidad por parte de los panelistas para cada tratamiento del melón, durante el tiempo del ensayo.

\section{DISCUSIÓN}

La pulpa de melón es ligeramente ácida; con un valor promedio de $\mathrm{pH}$ de 5,8, dicho valor obtenido se encuentra por debajo de los descritos por Raybaudi et al. (9) y la FDA (10) esta última establece un rango de 6,13 a 6,53; y similar a los reportados por Álvarez et al. (11) y por Arruda et al. (12), lo anterior permite deducir que la variación del $\mathrm{pH}$ en esta fruta puede estar relacionada con el tipo de suelo donde fue cultivado y la época del año. En este sentido, Laínez y Krarup (13) expresan que las variaciones en las condiciones físico-químicas de los melones se pueden asociar con factores ambientales y especialmente, al manejo del cultivo. Ramírez et al, (6) afirman que la mayoría de las frutas tropicales tiene un $\mathrm{pH}$ entre 2,6 y 5,8 , rango en el cual se encuentran los valores obtenidos para la pulpa de melón. Desde el punto de vista tecnológico, el pH en las frutas es un parámetro muy importante en el control del desarrollo de poblaciones de microorganismos, responsable de la actividad de sistemas enzimáticos, en los procesos de clarificación de jugos y bebidas, en la estabilidad de los mismos y de otros productos elaborados a partir de frutas; tales

\section{TABLA 2}

Evolución del pH del melón a través del tiempo.

\begin{tabular}{cccc}
\hline Días & $T_{0}$ & $T_{1}$ & $T_{2}$ \\
0 & $5,95 \pm 0,01 a$ & $5,95 \pm 0,01 \mathrm{a}$ & $5,95 \pm 0,01 \mathrm{a}$ \\
3 & $6,11 \pm 0,00 \mathrm{a}$ & $5,97 \pm 0,01 \mathrm{~b}$ & $5,96 \pm 0,01 \mathrm{~b}$ \\
6 & $6,16 \pm 0,01 \mathrm{a}$ & $5,98 \pm 0,01 \mathrm{~b}$ & $5,97 \pm 0,01 \mathrm{~b}$ \\
9 & $6,22 \pm 0,01 \mathrm{a}$ & $6,12 \pm 0,01 \mathrm{~b}$ & $5,98 \pm 0,01 \mathrm{c}$ \\
12 & $6,20 \pm 0,01 \mathrm{a}$ & $6,08 \pm 0,01 \mathrm{~b}$ & $5,97 \pm 0,01 \mathrm{c}$ \\
15 & $6,18 \pm 0,01 \mathrm{a}$ & $6,06 \pm 0,01 \mathrm{~b}$ & $5,94 \pm 0,01 \mathrm{c}$ \\
\hline
\end{tabular}

*** a,b,c valores con letras diferentes en una misma fila difieren significativamente $p<0,05$.

*** T0: tratamiento en blanco sin recubrimiento, T1: tratamiento con recubrimiento con almidón (5 mL), T2: tratamiento con recubrimiento con almidón (10 mL).

\section{TABLA 3}

Evolución de la acidez (\%) a través del tiempo.

\begin{tabular}{cccc}
\hline Días & $\mathrm{T}_{0}$ & $\mathrm{~T}_{1}$ & $\mathrm{~T}_{2}$ \\
0 & $0,10 \pm 0,00 \mathrm{a}$ & $0,10 \pm 0,00 \mathrm{a}$ & $0,10 \pm 0,00 \mathrm{a}$ \\
3 & $0,09 \pm 0,00 \mathrm{c}$ & $0,10 \pm 0,00 \mathrm{~b}$ & $0,10 \pm 0,00 \mathrm{a}$ \\
6 & $0,09 \pm 0,00 \mathrm{c}$ & $0,10 \pm 0,00 \mathrm{~b}$ & $0,10 \pm 0,00 \mathrm{a}$ \\
9 & $0,08 \pm 0,00 \mathrm{c}$ & $0,09 \pm 0,00 \mathrm{~b}$ & $0,10 \pm 0,00 \mathrm{a}$ \\
12 & $0,08 \pm 0,00 \mathrm{c}$ & $0,09 \pm 0,00 \mathrm{~b}$ & $0,10 \pm 0,00 \mathrm{a}$ \\
15 & $0,08 \pm 0,00 \mathrm{c}$ & $0,09 \pm 0,00 \mathrm{~b}$ & $0,10 \pm 0,01 \mathrm{a}$ \\
\hline
\end{tabular}

*** $a, b, c$ valores con letras diferentes en una misma fila difieren significativamente $p<0,05$

*** T0: tratamiento en blanco sin recubrimiento, T1: tratamiento con recubrimiento con almidón (5 mL), T2: tratamiento con recubrimiento con almidón (10 mL).

\section{TABLA 4}

Determinación de los sólidos solubles ( ${ }^{\circ}$ Brix).

\begin{tabular}{cccc}
\hline Días & $T_{0}$ & $T_{1}$ & $T_{2}$ \\
0 & $9,41 \pm 0,08 a$ & $9,41 \pm 0,08 a$ & $9,41 \pm 0,08 a$ \\
3 & $9,73 \pm 0,09 a$ & $9,30 \pm 0,05 b$ & $9,30 \pm 0,03 b$ \\
6 & $10,14 \pm 0,07 a$ & $9,51 \pm 0,07 b$ & $9,46 \pm 0,08 b$ \\
9 & $11,11 \pm 0,07 a$ & $10,1 \pm 0,09 b$ & $9,53 \pm 0,08 c$ \\
12 & $10,46 \pm 0,05 a$ & $9,61 \pm 0,07 b$ & $9,40 \pm 0,07 c$ \\
15 & $9,98 \pm 0,02 a$ & $9,19 \pm 0,06 c$ & $9,36 \pm 0,70 b$ \\
\hline
\end{tabular}

*** a,b,c valores con letras diferentes en una misma fila difieren significativamente $p<0,05$.

*** T0: tratamiento en blanco sin recubrimiento, T1: tratamiento con recubrimiento con almidón (5 mL), T2: tratamiento con recubrimiento con almidón (10 mL). 
como en la producción de jalea y mermelada cuya firmeza, color y flavor están determinados por la concentración de iones hidrógenos (6).

En lo referente al contenido de acidez se obtuvo un valor promedio de 0,10, similar a lo reportado por Álvarez et al. (11) y por Mosca et al. (14), igualmente se encuentra dentro de lo reportado por Cordeiro et al. (15), quienes obtuvieron valores de acidez entre 0,06 y 0,11 g de ácido cítrico/100 g de producto.

Con relación a los sólidos solubles, se encuentra dentro del rango reportado por Molina (16), quien toma como criterio para la cosecha de los melones aquellos que tengan un contenido de sólidos de 8 a $14{ }^{\circ} \mathrm{Brix}$, se puede considerar a esta fruta como comerciable.

En relación al diseño de los biorecubrimientos se evidenció un incremento del $\mathrm{pH}$ posiblemente se fundamenta en la maduración de las frutas. Los ácidos orgánicos de reserva presentes en las vacuolas, son transformados por la célula a azúcares que posteriormente son utilizados para la respiración celular, lo que ocasiona una disminución de la acidez del medio y con ello un aumento del pH (1).

Con respecto a los sólidos solubles, el mejor tratamiento fue el $T_{2}$, éste presentó la menor variación de sólidos solubles en los 15 días de conservación, debido al efecto de la cobertura de almidón modificado. En este sentido, Rojas et al. (17) señalan que una de las funciones que tienen los recubrimientos comestibles, aplicados en frutas cortadas, es la de producir una atmósfera controlada en ellas, que regula el transporte de gases, lo cual hace más lento el metabolismo de las frutas y en consecuencia la síntesis de compuestos azucarados. Resultados similares fueron reportados por Álvarez et al.(11), el cual evaluó el efecto de un recubrimiento comestible a base de gelatina en melón mínimamente procesado en el cual los ${ }^{\circ}$ Brix presentaron un comportamiento más estable comparado con un tratamiento control sin recubrimiento.

Posiblemente la marcada disminución de la acidez se debe a que las frutas al ser sometidas a estrés físico aumentan su actividad metabólica. En este orden de ideas, el descenso

\section{TABLA 5}

Porcentaje de pérdida de peso (\%) de los diferentes tratamientos.

\begin{tabular}{cccc}
\hline Días & $T_{0}$ & $T_{1}$ & 0 \\
0 & 0 & 0 & $0,69 \pm 0,00 c$ \\
3 & $4,15 \pm 0,00 \mathrm{a}$ & $1,98 \pm 0,00 \mathrm{~b}$ & $2,99 \pm 0,00 \mathrm{c}$ \\
6 & $5,58 \pm 0,02 \mathrm{a}$ & $4,80 \pm 0,04 \mathrm{~b}$ & $5,17 \pm 0,00 \mathrm{c}$ \\
9 & $7,37 \pm 0,11 \mathrm{a}$ & $7,05 \pm 0,00 \mathrm{~b}$ & $5,80 \pm 0,00 \mathrm{c}$ \\
12 & $10,86 \pm 0,05 \mathrm{a}$ & $7,56 \pm 0,03 \mathrm{~b}$ & $6,80 \pm 0,00 \mathrm{c}$ \\
\hline
\end{tabular}

*** a, b, c Superíndices diferentes en una misma fila difieren significativamente $p<0,05$

*** T0: tratamiento en blanco sin recubrimiento, T1: tratamiento con recubrimiento con almidón ( $5 \mathrm{~mL}$ ), T2: tratamiento con recubrimiento con almidón (10 mL).

\section{FIGURA 1}

Porcentaje de aceptación del melón troceado.

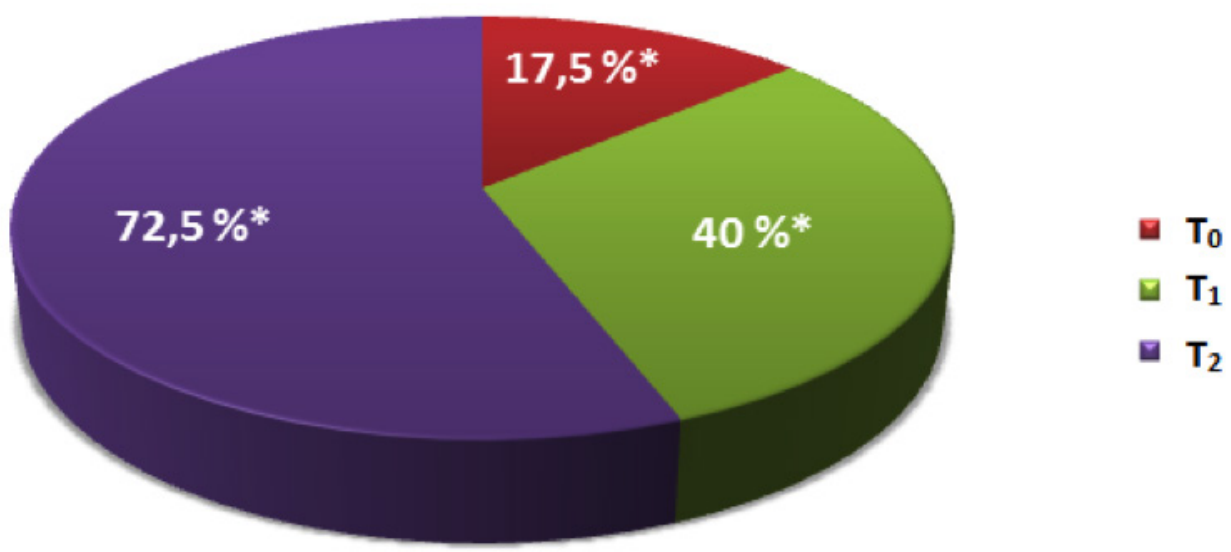

* Representa la cantidad de panelistas (en porcentaje) que calificaron la muestra con una puntuación igual o mayor que 4. 
de la acidez es debido a la actividad metabólica que experimentan las frutas durante la maduración, ya que en estas condiciones se originan actividades enzimáticas las cuales establecen una complicada red de cambios metabólicos que se traslapan y acoplan, lo que da origen a la conversión de los ácidos orgánicos de reserva de las frutas en azúcares que serán consumidos durante la respiración celular (18). Los ácidos pueden considerarse como una reserva energética de la fruta, por consiguiente es de esperar que su contenido decline en el periodo de actividad metabólica máxima durante el curso de la maduración. Por lo general la madurez presume un descenso de la acidez; de esta forma la relación azucares/ácidos aumenta durante la maduración de la mayor parte de las frutas (6).

La pérdida fisiológica de peso de los frutos aumentó con el tiempo de almacenamiento en todos los tratamientos como consecuencia de la transpiración. Los frutos tratados con los recubrimientos comestibles tuvieron menor pérdida de peso que el control al final del tiempo de conservación, siendo el recubrimiento de almidón $10 \mathrm{~mL}\left(T_{2}\right)$ el más efectivo $(p<0,05)$, perdiendo $6.8 \%$; por el contario, el tratamiento "blanco" (sin recubrimiento, T0) fue el que presentó mayor pérdida de peso con una variación del 13,8\%, valor similar fue reportado por Aguayo et al. (19), quien en su estudio de procesado en fresco del melón amarillo, alcanzó una pérdida del 13\%. Los productos procesados en fresco son mucho más vulnerables a la pérdida de agua, puesto que no poseen ninguna barrera para protegerse frente a la deshidratación. La corteza o piel en muchos casos cérea, ha sido eliminada y, evidentemente, convierte al producto procesado en altamente perecedero. Además, que al pelarlos se exponen directamente los tejidos internos a la atmósfera, aumentando drásticamente la velocidad de evaporación del agua $(1,2)$. El ensayo sensorial demostró que $T_{2}$ presentó una aceptabilidad, destacando que el almidón modificado mejora sus propiedades y su desempeño como recubrimiento sobre el melón. Por lo tanto el biorecubrimiento de melón con almidón de ñame modificado es una excelente alternativa para detener el deterioro del alimento, mantener la integridad estructural y mejorar las propiedades del melón.

\section{CONCLUSIONES}

Se confirma la efectividad de utilizar recubrimiento comestible a base de almidón modificado como una alternativa para la conservación del melón mínimamente procesado debido a que presentó una mayor aceptabilidad por parte de los panelistas, además presentó la menor variación de las propiedades fisicoquímicas.

\section{RESUMEN}

Los recubrimientos se definen como productos comestibles que forman una fina capa sobre el alimento y se caracterizan por que constituyen una barrera semipermeable a los gases y al vapor de agua que retrasa el deterioro del alimento, mejoran las propiedades mecánicas, ayudan a mantener la integridad estructural del producto que envuelven, ayudan a retener compuestos volátiles y pueden actuar como vehículo de aditivos alimentarios. Se evaluó el desempeño de los biorecubrimientos sobre el melón mediante la determinación de propiedades sensoriales y fisicoquímicas. Los resultados muestran que todas las variables están significativamente influenciadas por la biomolécula empleada "almidón modificado", observando un desempeño favorable en los biorecubrimientos comestibles.

Palabras clave: Biorecubrimiento, Cucumis melo L, almi- dón modificado, anhidro dodecenil succínico.

Agradecimientos: Los autores agradecen al SENA por la financiación del proyecto, al Centro de Comercio y Servicios de la regional Bolívar, asimismo a la Universidad de Cartagena por facilitar espacio y tiempo de los investigadores.

\section{BIBLIOGRAFÍA}

1. Vázquez-Ovando JA, Adriano-Anaya ML, Méndez-De León $R$, Salvador-Figueroa M. Preparation and physical characterization of composite chitosan-based biofilms. Quehacer Científico en Chiapas. 2013; 8 (2): 26 - 34.

2. Saavedra $N$, Algecira NA. Evaluation of edible films of cassava starch and isolated soy protein in the preservation of strawberries. NOVA - Publicación Científica Ciencias Bioméd. 2010; 8 (14): 121 - 240.

3. Park H. Development of advanced edible coatings for fruit. Trend food Sci Technol. 1999; 10: 254 - 60.

4. Morón FJ, Déborah P, Nodarse M. Assessment of scientific evidence recommending Annona muricata $L$. (soursop tree) for cancer prevention or treatment. Rev Cubana Plantas Medicinales. 2010; 15(3): 169-18.

5. AOAC. Official methods of analysis William Horwitz. Washington D.C: Assoc Analyti Chem; 1990.

6. Ramírez R, Arenas L, Acosta K, Yamarte M, Sandoval L. Effect of scalded on the nutritional quality pulp soursop (Annona muricata L.). Rev Iberoam Tecnol Postcosecha, 2012; 13(1): 48-57.

7. Ávila $R$, Pérez $M$, Giménez $A$, Hernández E. Soursop: A healthy raw material for the food and beverages industry. REDIP. UNEXPO. VRB. Venezuela. 2012; 2: 134-42.

8. Andrade RD, Ortega FA, Montes EJ, Torres $R$, Pérez OA, Castro M, Gutiérrez LA. Physicochemical and rheological characterization of guava pulp (Psidium guajava L.) varieties Hybrid Klom Sali, Puerto Rico, D14 and Red. Vitae, 2009; 16(1): 13-8.

9. Raybaudi RM, Soliva-Fortuny $R$, Martín-Belloso O. Use of antimicrobial agents for the conservation of fresh and fresh-cut fruits. In: González-Aguilar GA, Gardea AA, Cuamea-Navarro F. (Eds.), Microbiological quality assurance. CIAD, Hermosillo, Sonora, México. 2006; 2: 15-21.

10. FDA (Food and drug administration). approximate $\mathrm{pH}$ of foods and food products. 2003.

11. Álvarez C, Fermín N, García J, Peña E, Martínez A. Evaluation of the effect of applying an edible coating in melons (Cucumis melo L., var. cantaloupe) cut and stored in refrigeration. Saber, Universidad de Oriente, Venezuela. 2013. 25(2): 218-26.

12. Arruda M, Jacomino A, Fillet M, Gallo C, Moretti C. Conservation of minimally processed net melon under active modified atmosphere. Ciênc Tecnol Aliment Campinas. 2004. 24(1):53-8.

13. Laínez D, KraruP C. Pre- and post-harvest characterization of two reticulate melon cultivars of the Oriental type (Cucumis melo Group Catalupensis). Cienc Inv Agr. 2008; 35(1):59-66.

14. Mosca J, Piza I, Lima G. Biochemical markers for postharvest maturity in three melon cultivars. Hortic Bras. 2001. 19(1):210-15.

15. Cordeiro A, Wilane $R$, Arraes M, Elesbão A, Moreira M, Machado P. Effect of type of cutting on the physical chemical and microbiological characteristics of 'Cantaloupe' melon (Cucumis melo L. Hybrid hy-Mark) minimally processed. Ciên Agrotec. 2007: 31(4):1095-101. 
16. Ugás $R$, Siura S, Delgado F, Casas A, Toledo J. Vegetables program. Universidad Nacional Agraria La molina. Lima, Perú. 2000.

17. Rojas M, Raybaudi R, Soliva R, Avena F, Mchugh T, Martín $O$. Apple puree-alginate edible coating as carrier of antimicrobial agents to prolong shelf life of fresh-cut apples. Postharvest Biol Technol. 2007. 45 (2):254-64.

18. Baeza R. Comparison of technologies to control the physi- ological, biochemical and nutritional changes of fresh cut fruit. Master Work. College of Agriculture. Food Science Graduate Program. Manhattan, Kansas. 2007. Date of access: 04 April 2016. Available in: http://krex.k-state. edu/dspace/handle/2097/494

19. Aguayo E. \& Artés F. 2001. "Processed in the melon cool" yellow "and evolution of its sensory quality. Rev Alimentaria. 2001. 328: 87-92. 